\title{
A Pilot Clinical Trial of Radioprotective Effects of Curcumin Supplementation in Patients with Prostate Cancer
}

Jalal Hejazi ${ }^{1}$, Reza Rastmanesh ${ }^{1 *}$, Forough-Azam Taleban ${ }^{1}$, Seyed-Hadi Molana² and Golamreza Ehtejab²

${ }^{1}$ Department of Clinical Nutrition and Dietetics, Faculty of Nutrition Sciences and Food Technology, National Nutrition and Food Technology Research Institute, Shahid Beheshti University of Medical Sciences, Tehran, Iran

${ }^{2}$ Department of Radiation Oncology, Beasat Hospital, Armey Medical Sciences University, Tehran, Iran

\begin{abstract}
Background: Patients with prostate cancer who accede to radiation therapy usually experience several side effects and these toxicities are sometimes dose limiting. Some previous in vitro and in vivo studies have proposed a radioprotective role for curcumin, the yellow pigment of turmeric.
\end{abstract}

Objectives: The purpose of this investigation was to assess the radioprotective effects of curcumin supplementation in patients with prostate cancer.

Methods: Forty prostate cancer patients undergoing external beam radiotherapy (EBRT) were randomly assigned to curcumin group, taking $3 \mathrm{~g} / \mathrm{d}$ curcumin $(6 \times 500 \mathrm{mg}$ capsules of BCM95 $\mathrm{n}=20)$, or placebo group $(n=20)$. Quality of life was assessed by the Persian version of the European Organization for Research and Treatment of Cancer prostate cancer-specific quality of life questionnaire (QLQ-PR25).Analysis of covariance was used to compare radiotherapy related symptoms between groups following the intervention, adjusted for baseline symptoms.

Results: No differences in urinary symptoms, bowel symptoms, treatment related symptoms and sexual activity were observed between the curcumin and placebo groups before the intervention. The change in urinary symptoms across the 20-week period differed significantly between groups $(p=0.011)$ and patients in the curcumin group experienced much milder urinary symptoms compared with the placebo group. No group differences were observed in any other domain of the QLQ-PR25.

Conclusions: Curcumin can confer radioprotective effect in patients with prostate cancer who undergo radiation therapy through reducing the severity of radiotherapy related urinary symptoms. However supplementation with $3 \mathrm{~g} /$ day curcumin could not reduce the severity of bowel symptoms or other treatment related symptoms.

Keywords: Prostate cancer; Curcumin; Quality of life; Radioprotective

\section{Introduction}

Prostate cancer is the second most incident cancer among male population worldwide. It is the second leading cause of cancer death in American men [1]. There is no exact statistics on prostate cancer prevalence in Iran; however an estimation of 5 per 100,000 and 9 per 100,000 has been reported by two investigators $[2,3]$. A possible reason for this lower incidence comparing with many other countries is that there are no national programs for screening of prostate cancer in Iran.

Radiation therapy by itself or along with surgery and hormone therapy are the main treatments for prostate cancer [4]. However ionizing radiation can also have a harmful effect on healthy body tissues. Patients with prostate cancer who accede to radiation therapy usually experience some degrees of sexual dysfunction, gastrointestinal disorders and urinary tract problems [5].These toxicities are known to be dose-limiting, [6] and because higher radiation doses for patients with clinically localized prostate cancer are now considered standard of care [7], finding ways to decrease symptoms burden is crucial.

Recently some in vitro and in vivo studies showed radiosensitizing and radioprotective effects by some phytochemicals [8]. One of these phytochemicals is curcumin. Curcumin is the yellow coloring agent in the spice, turmeric. Turmeric has been long used as a spice and food additive in Iranian cuisines. Curcumin has been reported to protect various study systems, in vitro and in vivo, against the deleterious effects induced by ionizing radiation and to enhance the effect of radiation
[9-11]. Therefore, curcumin has the potential to be very useful during radiotherapy of prostate cancer.

The present pilot double blinded placebo controlled clinical trial, is designed to assess radioprotective effects of curcumin supplementation in patients with prostate cancer. To the best of our knowledge this is the first reported clinical trial testing curcumin supplement to test quality of life in patients with prostate cancer.

\section{Materials and Methods}

Between March 2011 and March 2013, all patients recently diagnosed with localized prostate cancer at the Department of Oncology at Besat Hospital were assessed for eligibility. Patients referred to local

*Corresponding author: Reza Rastmanesh, Department of Clinical Nutrition and Dietetics, Faculty of Nutrition Sciences and Food Technology, National Nutrition and Food Technology Research Institute, Shahid Beheshti University of Medical Sciences, Tehran- 4739, Iran, Tel: +9821-22357484 (ex 253); Fax: +982122360660; E-mail: rezar@sbmu.ac.ir

Received August 27, 2013; Accepted October 07, 2013; Published October 09 2013

Citation: Hejazi J, Rastmanesh R, Taleban FA, Molana SH, Ehtejab G (2013) A Pilot Clinical Trial of Radioprotective Effects of Curcumin Supplementation in Patients with Prostate Cancer. J Cancer Sci Ther 5: 320-324. doi:10.4172/19485956.1000222

Copyright: $\odot 2013$ Hejazi J, et al. This is an open-access article distributed under the terms of the Creative Commons Attribution License, which permits unrestricted use, distribution, and reproduction in any medium, provided the original author and source are credited. 
curative radiotherapy with external beam radiotherapy (EBRT), in combination with hormone ablation, were invited to participate in the study (ClinicaltiralsNCT01917890). Adenocarcinoma of the prostate must be histologically confirmed on biopsy. All patients were with a life expectancy greater than 5 years. No metastatic disease must be detected during physical examination, standard radiography, bone scan, and magnetic resonance spectroscopy (MRS). Additional inclusion criteria were no prior hormone therapy, radiotherapy or systemic treatment for prostate cancer and no other malignancy. The exclusion criteria were clinical stage T3 or T4, Gleason score $\geq 8$, serum PSA $\geq 20 \mathrm{ng} / \mathrm{mL}$, other prior surgery for prostate cancer, concurrent participation in another clinical trial which would require approval upon entry to this trial, gastrointestinal disorders such as inflammatory bowel disease, reflux and peptic ulcers and any adverse reaction to curcumin. Forty five of 78 eligible patients agreed to participate and 40 patients completed the study (Figure 1). All patients gave their written informed consent prior to participation. The study was approved by the ethical committee of National Nutrition and Food Technology Research Institute.

EBRT was given as daily fractions of $2 \mathrm{~Gy}$ to achieve a total dose of 74 Gy (5 times a week for about 8 weeks). Data were collected 1 week prior to radiotherapy onset, at the same time as randomization and intervention onset, and 3 months after radiotherapy and intervention completion.

\section{Randomization}

Patients were randomly assigned to either the curcumin group (CG, $n=43$ ) or placebo group (PG, $n=42$ ) (Figure 1). Randomization was performed by administrative personnel outside the research project in a double-blind fashion. Random assignment was based on a computer-generated randomization list obtained using blocks of size 4 .

\section{Intervention}

Curcumin capsules (BCM95 (Biocurcumin)) and their placebos

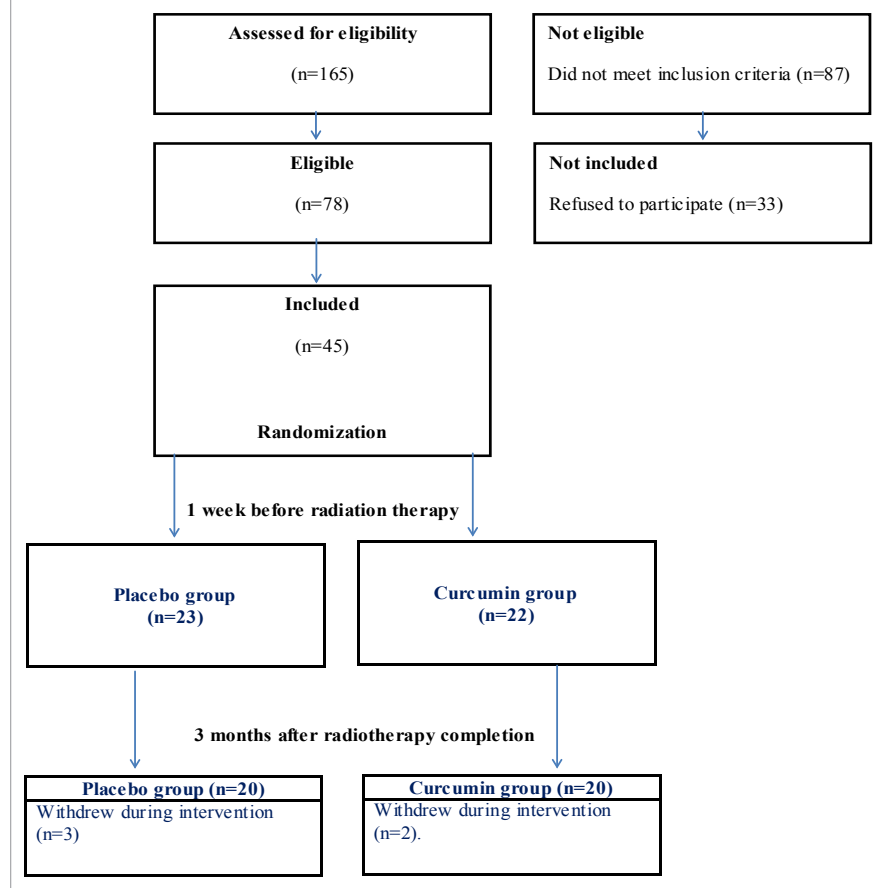

Figure1: Flow of participants throughout trial. were obtained as a generous donation from Arjuna Natural Extracts Ltd. Kerela, India, in $500 \mathrm{mg}$ capsule form. Each curcumin capsule contained $440 \mathrm{mg}$ curcuminoids ( $347 \mathrm{mg}$ curcumin, $84 \mathrm{mg}$ desmethoxycurcumin, and $9 \mathrm{mg}$ bisdesmethoxycurcumin) and essential oil of turmeric $38 \mathrm{mg}$ and each placebo capsule contained $500 \mathrm{mg}$ roasted rice flour.

Patients in each group took 3 grams of curcumin or placebo (as $6 \times$ $500 \mathrm{mg}$ capsules, 2 capsules with each meal) since 1 week before onset of radiotherapy until completion of their radiotherapy.

All patients were advised to avoid any changes in their usual dietary habits during intervention period.

\section{Evaluation during study}

A complete history [pathologic confirmation of malignancy, disease staging, prior therapy/surgery, and prior response(s)] and a physical examination, as well as blood tests (including a complete blood count and PSA) were done at baseline by a trained physician.

All patients were referred to Babak imaging center for MRS. MRI/ MRSI studies were performed on a 1.5 Tesla Sigma Horizon scanner (GE, Milwaukee, WI) using a combined pelvic phased array and endorectal coil (Medrad, Indianola, PA). MRSI data were obtained and processed using software developed at the University of California in San Francisco.

Patients were weighed in light clothing and without shoes using a scale (Seca, Hamburg, Germany) and their height, measured with a stadiometer (Holtain Ltd, Crymych, United Kingdom), was used to calculate Body Mass Index (BMI).

A validated semi-quantitative food frequency questionnaire (FFQ) [12] was used to evaluate dietary intake of patients. For the extraction of polyphenols intake from foods in FFQ, the phenol-Explorer database was used. Phenol-Explorer database contains data on the content of 502 polyphenols in 452 foods [13].

The European Organization for Research and Treatment of Cancer (EORTC) prostate cancer-specific quality of life questionnaire module (QLQ-PR25) was used to assess urinary, sexual, and bowel function [14]. The questionnaire was translated from English into Persian and then translated back from Persian into English by a researcher fluent in both languages. Reliability $(\mathrm{r}=0.85)$ and construct validity (Cronbach alpha $>0.7$ ) had been determined in our pilot study with 41 patients. The test-retest reliability was satisfactory (Pearson $\mathrm{r}=0.61$ to 0.93 for individual items and 0.85 for total score). The research team, several university faculty members, and a physician reviewed the pilot questionnaire to establish face validity and trustworthiness. The questionnaire was revised appropriately based on their feedback. Items were combined into several scales (from 1 to 100) according to EORTC rules. For the global health and function scales, a high score signaled a better QoL; for the symptom scales, a high score was indicative of a poorer QoL.

All the above evaluations were done at baseline ( 1 week before radiotherapy onset) and 3 months after radiotherapy completion.

\section{Statistical analyses}

Statistical analyses were performed using SPSS 19.0 (SPSS, Chicago, IL). All analyses were conducted on an intention-to-treat basis. All p-values were two-tailed and the level of statistical significance was set at $p<0.05$. Between-group differences at baseline were analyzed using the chi-square test and the independent t-test. 
Analysis of covariance (ANCOVA) adjusting for baseline was used to evaluate the effect of the intervention on side effects using data from QLQ-PR25.

\section{Results}

The two randomized groups were well balanced with no significant differences in characteristics at baseline (Table 1). All the patients who completed the study ( 40 out of 45 patients), took all their supplements and because we used face to face interview for completion of questionnaires, there was no missing answer.

There was no significant difference between the 2 groups in terms of 4 subclasses of QLQ-PR25 (urinary symptoms, bowel symptoms, treatment-related symptoms and sexual functioning) at baseline (Table 2). The change in urinary symptoms across the 20 -week period differed significantly between groups ( $\mathrm{p}=0.011$, Table 2 ). Following the intervention, participants in the curcumin group had significantly lower increase in urinary symptoms comparing with placebo group. No group differences were observed in any other domain of the QLQPR25.

Figure 2 shows the number of patients with worsening different urinary symptoms 3 month after radiotherapy. As it is shown, patients in curcumin group experienced much milder problems in terms of most of the urinary symptoms specially urinate frequency during the day and limitation of their daily activities. Only 2 of our participants were wearing an incontinence aid so this part of questionnaire was not considered in our analysis.

\section{Discussion}

To our knowledge this is the first randomized controlled trial investigating the radioprotective effect of curcumin in prostate cancer patients. The primary finding of this analysis was that $3 \mathrm{~g} /$ day curcumin supplement can reduce the severity of radiotherapy related urinary symptoms in patients with prostate cancer.
The3g dose of curcumin was chosen based on the results of an in vitro study which has shown that curcumin in the concentration of $2 \mu \mathrm{M}$ can confer radiosensitizing activity on human prostate cancer cells and enhance apoptosis [15]. Also a clinical trial has shown that by taking $2 \mathrm{~g}$ of BCM95the concentration of $2 \mu \mathrm{M}$ is achievable in plasma [16]. Moreover according to the previous studies, this dose is absolutely safe [17]. However this dose of curcumin did not appear to be effective at reducing the severity of bowel symptoms or other treatment related symptoms. In the case of sexual function, although participants in curcumin group had better condition compared with placebo group, this difference did not reach statistical significance. Hormone deprivation therapy of the patients can be a possible confounding factor which can mask the true effect of curcumin, however it was a part of patients' treatment program and could not be omitted. The non dose-finding design of this clinical trial could be seen as a limitation because the real effective dose of curcumin for these symptoms cannot be discerned and higher doses may have better protective effects.

In a recent clinical trial supplementation with $6 \mathrm{~g} / \mathrm{d}$ of curcumin reduced the severity of radiation dermatitis in breast cancer patients [18]. Also, several in vitro and in vivo studies which were reviewed by Goel et al. have indicated that curcumin has the potential to be used as a radioprotective agent [19].

The majority of the acute urinary dysfunction caused by EBRT relates to inflammation and mucosal loss at the bladder neck and within the prostate and prostatic urethra [5]. Curcumin is a well-known and strong anti-inflammatory and anti-oxidant agent. The mechanisms responsible for the protective effect of curcumin on urinary were not specifically examined in this investigation. However some mechanisms have been proposed for the radioprotective effect of curcumin. In an in vivo study it had been shown that curcumin confers its radioprotective effect through decreasing gene expression of inflammatory (IL-1, IL-6, IL-18, TNFa, and lymphotoxin- $\beta$ ) and fibrogenic cytokine (TGF $\beta$ ) [20]. Also it has been reported that curcumin scavenges free radicals, increases antioxidant status, inhibits lipid peroxidation, and

\begin{tabular}{|c|c|c|c|}
\hline Baseline variables & CG $(n=20)$ & $P G(n=20)$ & $P$ \\
\hline Age (years) & $69.58 \pm 8.08$ & $71.85 \pm 8.33$ & 0.394 \\
\hline Height (cm) & $167.48 \pm 6.09$ & $167.63 \pm 5.50$ & 0.935 \\
\hline Weight (kg) & $76.26 \pm 10.79$ & $75.53 \pm 12.28$ & 0.844 \\
\hline BMI $\left(\mathrm{kg} / \mathrm{m}^{2}\right)$ & $27.17 \pm 3.37$ & $26.81 \pm 3.68$ & 0.750 \\
\hline Married & $18(90)$ & 19(95) & 0.548 \\
\hline Gleason score & $6.50 \pm 0.97$ & $6.83 \pm 0.39$ & 0.288 \\
\hline PSA (ng/ml) & $12.98 \pm 7.09$ & $16.47 \pm 5.94$ & 0.130 \\
\hline Calorie intake (Kcal) & $2145.38 \pm 584.5$ & $2199.41 \pm 442.62$ & 0.744 \\
\hline Total polyphenol intake (mg) & $3315.27 \pm 2019.38$ & $2925.63 \pm 1065.67$ & 0.450 \\
\hline
\end{tabular}

Abbreviations: CG, curcumin group; PG, placebo group; BMI, body mass index; PSA, prostate specific antigen. Results presented as mean \pm s.d. or number of participants (percentage of participants)

Table1: Baseline characteristics of participants.

\begin{tabular}{|c|c|c|c|c|c|c|c|c|c|c|c|c|}
\hline & \multicolumn{4}{|c|}{1 week before radiation therapy onset } & \multicolumn{4}{|c|}{3 months after radiation completion } & \multicolumn{4}{|c|}{$\begin{array}{l}\text { Adjusted group differences in mean } \\
\text { change over } 20 \text { weeks }\end{array}$} \\
\hline & \multicolumn{2}{|c|}{ CG } & \multicolumn{2}{|c|}{ PG } & \multicolumn{2}{|c|}{ CG } & \multicolumn{2}{|c|}{ PG } & Mean & \multicolumn{2}{|c|}{$95 \% \mathrm{Cl}$} & $P$ value ${ }^{a}$ \\
\hline & Mean & SD & Mean & SD & Mean & SD & Mean & SD & & & & \\
\hline Urinary symptoms & 16.2 & 6.5 & 18.8 & 5.6 & 24.2 & 15.8 & 38.7 & 16.3 & -14.1 & -24.7 & -3.4 & 0.011 \\
\hline Bowel symptoms & 16.2 & 19.2 & 19.5 & 22.2 & 25.0 & 12.4 & 20.0 & 18.0 & 5.4 & -4.5 & 15.4 & 0.275 \\
\hline Treatment-related symptoms & 27.2 & 14.9 & 25.0 & 9.4 & 39.2 & 17.2 & 31.1 & 16.6 & 7.9 & -3.1 & 18.9 & 0.155 \\
\hline Sexual activity & 18.3 & 24.7 & 22.5 & 25.5 & 16.7 & 22.9 & 20.8 & 25.3 & -3.5 & -19.0 & 12.0 & 0.652 \\
\hline
\end{tabular}

Abbreviations: CG, curcumin group; PG, placebo group; ANCOVA, analysis of covariance; $\mathrm{Cl}$, confidence interval

aBetween-group change by ANCOVA adjusted for baseline values

Table2: Prostate cancer-specific quality of life. 


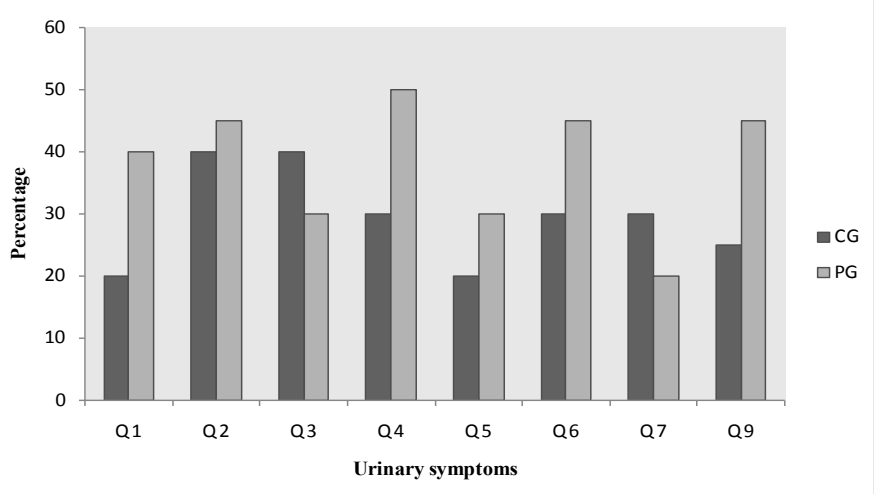

Figure 2: Percentage of men with worsening different urinary symptoms according to EORTC QLQ - PR25, three months after radiotherapy. Q1, urinate frequently during the day; $Q 2$, urinate frequently at night; $\mathrm{Q}$, have to hurry to get to the toilet; $\mathrm{Q} 4$, not enough sleep because urinate frequently at night; Q5, difficulty in going out because of the needed to be close to a toilet; Q6, unintentional release (leakage) of urine; Q7, having pain when urinating; Q9, limitation of daily activity because of urinary problems.

elevates glutathione S-transferases, glutathione peroxidase, superoxide dismutases, glutathione, and sulphydryl groups [21].

Another issue which makes curcumin supplementation during radiation therapy an interesting possibility is the fact that any exposure to radiation raises concern about the possibility of a second malignancy. A population based study of 17,845 prostate cancer patients compared secondary cancer incidence in patients treated with radical prostatectomy and radio therapy [22]. The results showed that the risk of rectal cancer, bladder cancer and lung cancer in patients treated with radiotherapy are significantly higher compared with those who underwent radical prostatectomy. Curcumin is a potent anticancer agent which suppresses all 3 stages of carcinogenesis: initiation, promotion, and progression [19] and can be a promising anti-cancer treatment. There is a common concern that using antioxidants during radiotherapy may reduce the efficacy of treatment by protecting tumor cells from radiation-induced cell death. Despite of controversies about the impacts of antioxidant supplementation during radiation therapy on its outcomes, some recent studies have reported promising effects $[23,24]$. In the present study MRI/MRS results and PSA levels of patients in CG and PG was not significantly differed, 3 months after intervention completion $(0.12 \pm 0.17$ and $0.13 \pm 0.06$, respectively). Thus it seems that at least in short term, curcumin does not have any unfavorable effect on treatment outcomes. Several in vitro studies have also proposed a radiosensitizing activity for curcumin by inhibition of radiation-induced elevation of growth factors, cytokines, cyclins, nuclear factor- $\kappa \mathrm{B}$ and tumor necrosis factor- $\alpha$ on tumor cells [21] and probably these mechanisms are blunting the radioprotective effects of curcumin in tumor cells. The study of molecular mechanisms of radioprotective effect of curcumin on normal tissues and its radiosensitization on tumor cells is on its way by our research team.

There are some limitations associated with this investigation including the small sample size and short duration of follow up. Larger clinical trials with different doses of curcumin are desirable to further confirm curcumin radioprotective effects in different organs. The questionnaire was pilot tested to establish construct validity, but the questionnaire cannot be declared reliable without test-retest analysis in other studies.

\section{Conclusion}

The present clinical trial has shown that curcumin can confer radioprotective effect in patients with prostate cancer who undergo radiation therapy through reducing the severity of radiotherapy related urinary symptoms which are of the most common side effects of radiation therapy [5]. However supplementation with $3 \mathrm{~g} /$ day curcumin could not reduce the severity of bowel symptoms or other treatment related symptoms. Further studies with larger sample sizes and higher doses of curcumin are required to further confirm radioprotective effects of curcumin on different organs.

\section{Acknowledgements}

This study was part of $\mathrm{PhD}$ dissertation by the first author and was supported by the research grants from National Nutrition and Food Technology Research Institute, Iran National Science Foundation, and research deputy of Shahid Beheshti University of Medical Sciences. We gratefully acknowledge Arjuna Natural Extracts Ltd for providing the curcumin capsules and placebos.

This study was funded by grants from National Nutrition and Food Technology Research Institute, Iran National Science Foundation, and research deputy of Shahid Beheshti University of Medical Sciences.

\section{References}

1. Jemal A, Siegel R, Xu J, Ward E (2010) Cancer statistics, 2010. CA Cancer J Clin 60: 277-300.

2. Mousavi SM, Gouya MM, Ramazani R, Davanlou M, Hajsadeghi N, et al (2009) Cancer incidence and mortality in Iran. Ann Oncol 20: 556-563.

3. Sadjadi A, Nooraie M, Ghorbani A, Alimohammadian M, Zahedi MJ, et al. (2007) The incidence of prostate cancer in Iran: results of a population-based cancer registry. Arch Iran Med 10: 481-485.

4. Nilsson S, Norlén BJ, Widmark A (2004) A systematic overview of radiation therapy effects in prostate cancer. Acta Oncol 43: 316-381.

5. Michaelson MD, Cotter SE, Gargollo PC, Zietman AL, Dahl DM, et al. (2008) Management of complications of prostate cancer treatment. CA Cancer J Clin 58: 196-213

6. Wahlgren T, Brandberg Y, Häggarth L, Hellström M, Nilsson S (2004) Healthrelated quality of life in men after treatment of localized prostate cancer with external beam radiotherapy combined with< sup> 192 ir brachytherapy: A prospective study of 93 cases using the EORTC questionnaires QLQ-C30 and QLQ-PR25. Int J Radiat Oncol Biol Phys 60: 51-59.

7. Zelefsky MJ, Levin EJ, Hunt M, Yamada Y, Shippy AM, Jackson A, et al. (2008) Incidence of late rectal and urinary toxicities after three-dimensional conformal radiotherapy and intensity-modulated radiotherapy for localized prostate cancer. Int J Radiat Oncol Biol Phys 70: 1124-1129.

8. Garg AK, Buchholz TA, Aggarwal BB (2005) Chemosensitization and radiosensitization of tumors by plant polyphenols. Antioxid Redox Signal 7 : 1630-1647.

9. Akpolat M, Kanter M, Uzal MC (2009) Protective effects of curcumin against gamma radiation-induced ileal mucosal damage. Arch Toxicol 83: 609-617.

10. Aravindan N, Madhusoodhanan R, Ahmad S, Johnson D, Herman T (2008) Curcumin inhibits NFkappaB mediated radioprotection and modulate apoptosis related genes in human neuroblastoma cells. Cancer Biol Ther 7: 569-576.

11. Khafif A, Hurst R, Kyker K, Fliss DM, Gil Z, et al. (2005) Curcumin: a new radiosensitizer of squamous cell carcinoma cells. Otolaryngol Head Neck Surg 132 317-321.

12. Mirmiran $P$, Esfahani FH, Mehrabi $Y$, Hedayati M, Azizi $F$ (2010) Reliability and relative validity of an FFQ for nutrients in the Tehran lipid and glucose study. Public Health Nutr 13: 654-662.

13. Pérez-Jiménez J, Neveu V, Vos F, Scalbert A (2010) Systematic analysis of the content of 502 polyphenols in 452 foods and beverages: an application of the phenol-explorer database. J Agric Food Chem 58: 4959-4969.

14. van Andel G, Bottomley A, Fosså SD, Efficace F, Coens C, et al. (2008) An international field study of the EORTC QLQ-PR25: a questionnaire for assessing the health-related quality of life of patients with prostate cancer. Eur J Cancer 44: 2418-2424. 
Citation: Hejazi J, Rastmanesh R, Taleban FA, Molana SH, Ehtejab G (2013) A Pilot Clinical Trial of Radioprotective Effects of Curcumin Supplementation in Patients with Prostate Cancer. J Cancer Sci Ther 5: 320-324. doi:10.4172/1948-5956.1000222

15. Chendil D, Ranga RS, Meigooni D, Sathishkumar S, Ahmed MM (2004) Curcumin confers radiosensitizing effect in prostate cancer cell line PC-3. Oncogene 23: 1599-1607.

16. Antony B, Merina B, lyer VS, Judy N, Lennertz K, et al. (2008) A Pilot Cross-Ove Study to Evaluate Human Oral Bioavailability of BCM-95CG (Biocurcumax), A Novel Bioenhanced Preparation of Curcumin. Indian J Pharm Sci 70: 445-449.

17. Cheng AL, Hsu CH, Lin JK, Hsu MM, Ho YF, et al. (2001) Phase I clinical trial of curcumin, a chemopreventive agent, in patients with high-risk or pre-malignant lesions. Anticancer Res 21: 2895-2900.

18. Ryan JL, Heckler CE, Ling M, Katz A, Williams JP, Pentland AP, et al. (2013) Curcumin for Radiation Dermatitis: A Randomized, Double-Blind, PlaceboControlled Clinical Trial of Thirty Breast Cancer Patients. Radiat Res 180: 3443.

19. Goel A, Aggarwal BB (2010) Curcumin, the golden spice from Indian saffron, is a chemosensitizer and radiosensitizer for tumors and chemoprotector and radioprotector for normal organs. Nutr Cancer 62: 919-930.
20. Okunieff P, Xu J, Hu D, Liu W, Zhang L, et al. (2006) Curcumin protects against radiation-induced acute and chronic cutaneous toxicity in mice and decreases mRNA expression of inflammatory and fibrogenic cytokines. Int J Radiat Onco Biol Phys 65: 890-898.

21. Jagetia GC (2007) Radioprotection and radiosensitization by curcumin. The Molecular Targets and Therapeutic Uses of Curcumin in Health and Disease: Springer: $301-320$

22. Bhojani N, Capitanio U, Suardi N, Jeldres C, Isbarn H, Shariat SF, et al. (2010) The rate of secondary malignancies after radical prostatectomy versus external beam radiation therapy for localized prostate cancer: a population-based study on 17,845 patients. Int J Radiat Oncol Biol Phys 76: 342-348.

23. Fuchs-Tarlovsky V (2012) Role of antioxidants in cancer therapy. Nutrition 29 15-21.

24. Margalit DN, Kasperzyk JL, Martin NE, Sesso HD, Gaziano JM, Ma J, et al (2012) Beta-carotene Antioxidant Use During Radiation Therapy and Prostate Cancer Outcome in the Physicians' Health Study. Int J Radiat Oncol Biol Phys 83: 28-32. 